\title{
New localities for Tylototriton uyenoi, T. panhai and T. anguliceps in Thailand with remarks on the southernmost distribution of the genus
}

\author{
AXEL HERNANDEZ ${ }^{1}$, DANIEL ESCORIZA ${ }^{2}$, PORRAWEE POMCHOTE ${ }^{3} \& \mathrm{MIAN} \mathrm{HOU}^{4}$ \\ ${ }^{1}$ Department of Environmental Sciences, Faculty of Sciences and Technics, University Pasquale Paoli of Corsica, Corte, \\ 20250, France \\ ${ }^{2}$ GRECO, Institute of Aquatic Ecology, University of Girona, Campus Montilivi, 17071 Girona, Spain \\ ${ }^{3}$ Department of Biology, Faculty of Science, Chulalongkorn University, Bangkok 10330, Thailand \\ ${ }^{4}$ College of Continuing Education, Sichuan Normal University, Chengdu, Sichuan, 610068, China \\ Corresponding author Email: hernandez.axel.1989@gmail.com
}

\section{INTRODUCTION}

$T^{1}$ he genus Tylototriton inhabits tropical and subtropical, moist broad-leaf forests close to waterbodies at moderate to high elevations between 1,250 and $1,900 \mathrm{~m}$ above sea level (m a.s.I.) in Thailand (Taylor, 1962; Wongratana, 1984; Nabhitabhata \& Chan-ard, 2005; Pomchote et al., 2008; Nishikawa et al., 2013; Hernandez, 2016a,b; Dowwiangkan et al., 2018). Three species were reported in the region, Tylototriton uyenoi, Tylototriton panhai and Tylototriton anguliceps (Nishikawa et al., 2013; Le et al., 2015). These crocodile newts are distributed throughout northern Thailand but their occurrence and ecological requirements are poorly known (Hernandez, 2015, 2016a,b; Hernandez et al., 2018). Tylototriton uyenoi occurs from the Dawana and the Daen Lao Hills and from the Phi Pan Nam to the Inthanon Range (Gerlach, 2012; Michaels, 2015; Hernandez, 2015, 2016a,b, 2017; Dowwiangkan et al., 2018). The Daen Lao Hills are a mountain network that extends to the southern Shan states and Karen Hills (Kayah and Kayin States bordering Mae Hong Son) in Myanmar. This mountain system is connected to the north-western mountains of Thailand where $T$. uyenoi can also occur (Hernandez, 2017). Tylototriton panhai inhabits mixed deciduous, dry dipterocarp, dry evergreen, and hill evergreen forests at elevations between 1,285 to $1,688 \mathrm{~m}$ a.s.l (Pomchote et al., 2008; Nishikawa et al., 2013; Hernandez, 2016a,b, 2017). Its presence is known from Phitsanulok, Uttaradit, Phetchabun and Loei Provinces in north-eastern Thailand, and east into Sainyabuli Province, Botene District, Laos (Wongratana, 1984; Nabhitabhata \& Chan-ard, 2005; Pomchote et al., 2008; Nishikawa et al., 2013; Hernandez, 2015, 2016a,b; Phimmachak et al., 2015). Tylototriton anguliceps inhabits evergreen forests at elevations of 1,300$1,800 \mathrm{~m}$ a.s.I in north-western Vietnam, northern Laos and north-eastern Thailand (Le et al., 2015; Phimmachak et al., 2015). Here we report four new records for these Tylototriton species in northern and north-eastern Thailand and we discuss their biogeographical preferences (Fig. 1).

\section{MATERIALS AND METHODS}

We conducted field work over a three-year period 2014-2016 and 2018 respectively, during the monsoon season (April to September) in north-western, northern and north-eastern Thailand.

Observations of the vegetation and habitat of salamanders were made during field trips both on sunny and on rainy days from approximately 08:00 $\mathrm{h}$ to 23:30 $\mathrm{h}$. We surveyed almost all types of habitat which included permanent and temporary streams but also ponds (including artificial reservoirs and irrigation canals), and surrounding terrestrial habitats, stumps, stones and litter. For identification purposes, we photographed using a digital camera (Sony Nex-5; Sony Ltd., Japan) each taxon observed, including plant and tree species. Coordinates, geographic and elevational data were collected in situ using a Global Positioning System (Garmin Montana 680; Garmin Ltd., Olahe, KS, USA) and located on maps. Water $\mathrm{pH}$ and temperature was recorded in situ using a Expresstech @ LCD PH Medidor Digital (Expresstech; Kingpow Company Limited; Hong-Kong; China). We also assessed the relative positions of the species on environmental gradients using principal component analysis (PCA). This allows us to visualise if these new localities are within the ecological range (95\% confidence ellipses) expected for these species (Hernandez et al., 2018). For this we followed the protocol of Hernandez et al. (2018), including the mean annual temperature $\left({ }^{\circ} \mathrm{C}\right)$, temperature seasonality, annual precipitation $(\mathrm{mm})$, and precipitation seasonality, from the WorldClim database (Hijmans et al., 2005). We also included a descriptor of the topography (index of terrain ruggedness) and the percentage of forest cover (Tuanmu \& Jetz, 2014). The variables were normalised before the PCA was implemented using the Paleontological Statistics package (PAST; Hammer, 2015).

\section{RESULTS}

We found T. uyenoi at Doi Mon Jong, Tak province, northwestern Thailand $\left(17^{\circ} 32^{\prime} 12.18^{\prime \prime} \mathrm{N}, 98^{\circ} 31^{\prime} 47.30^{\prime \prime}\right.$ E) $1,597 \mathrm{~m}$ 


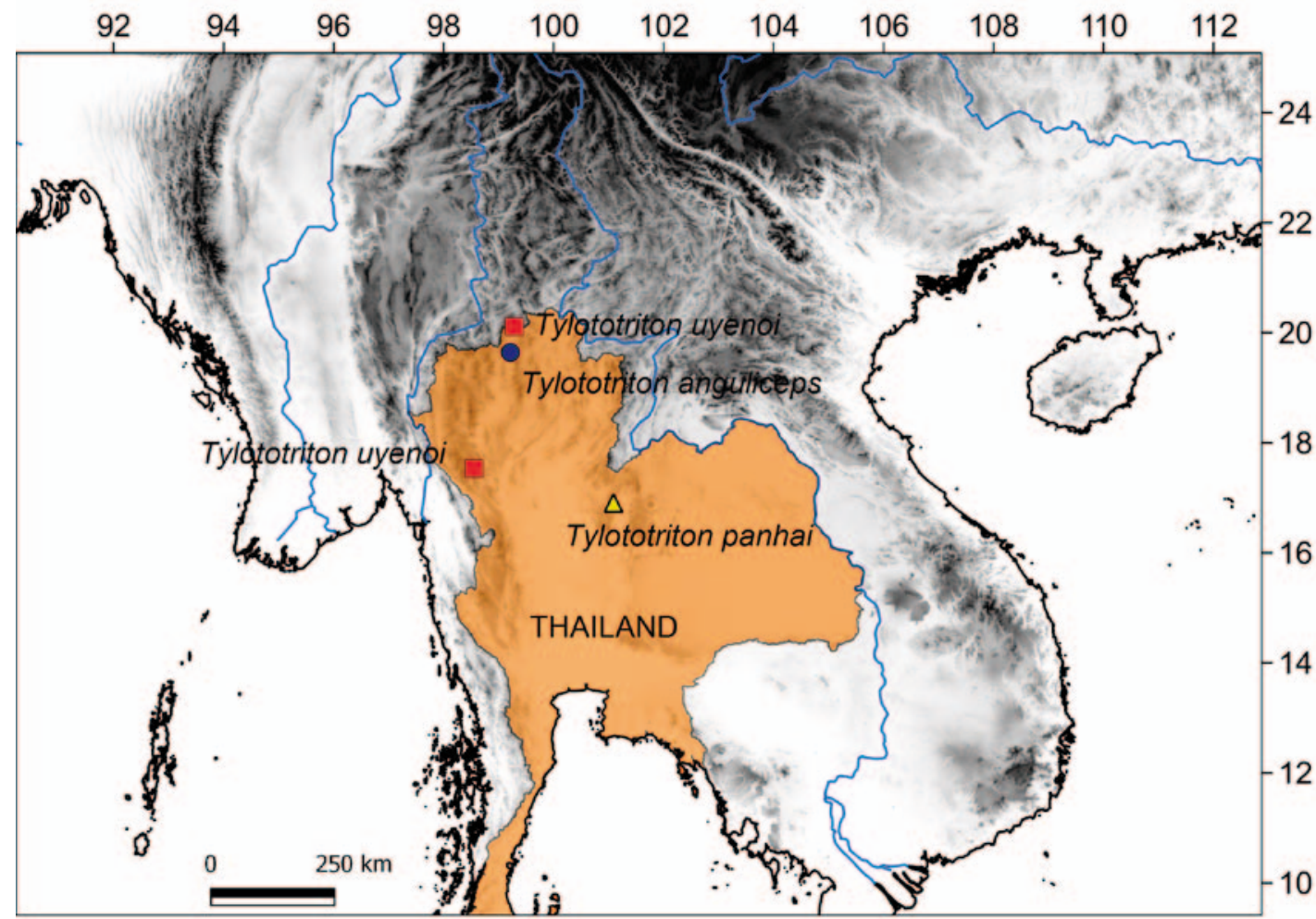

Figure 1. Map of Thailand (shaded orange) and neighbouring countries, showing the four new locations described in this study: Blue circle, T. anguliceps; Red squares, T. uyenoi; Yellow triangle, T. panhai

a.s.I. One adult male (Fig. 2A) was found near a small pond in a montane evergreen forest. Four eggs were also recorded from the same pond. They were deposited one by one in the submerged vegetation. The finding confirmed that this species lays eggs underwater like other species of the subgenus Tylototriton (Raffaëlli, 2013; Hernandez, 2016a,b). We found T. uyenoi at Doi Mak Lang, Mae Ai district, Chiang Mai province, northern Thailand $\left(20^{\circ} 6^{\prime} 47^{\prime \prime} \mathrm{N}, 99^{\circ} 15^{\prime} 37^{\prime \prime}\right.$ E) 1,456 m a.s.l. One adult male was observed under a large rotten tree in a hill evergreen forest dominated by Ostodes paniculata, Canarium bengalense and Polyspora axillaris (Fig. 2B). The air temperature was $21.5^{\circ} \mathrm{C}$ with a relative humidity of $76 \%$. This specimen was blackish-to brown in colour and showed robust parotoid glands, being similar to topotypic $T$. verrucosus. It measured a total length of $168 \mathrm{~mm}(\mathrm{TL})$.

Furthermore, on 12 July 2014, three adults of T. panhai were found at Phu Thap Boek, Lom Kao district, Phetchabun province, north-eastern Thailand $\left(16^{\circ} 54^{\prime} 23^{\prime \prime} \mathrm{N}, 101^{\circ} 5^{\prime} 14^{\prime \prime} \mathrm{E}\right)$ $1,663 \mathrm{~m}$ a.s.l. These individuals were observed in a small stream. One adult male measured $132 \mathrm{~mm}(\mathrm{TL})$ showing the typical traits of the "type III" (Fig. 3A; see Hernandez 2016a,b, 2017): it showed a dark reddish brown colouration with 1113 small reddish dorsolateral glandular warts (sometimes indistinct in adult specimens). These warts were placed on the dorsum, laterally, very close to each other. The shape of the head and the cephalic edges were also more angular than in other phenotypes. The vegetation around the stream included trees and giant perennial grasses of the genus Musa. The air temperature was $26.4{ }^{\circ} \mathrm{C}$, water temperature $20.2^{\circ} \mathrm{C}$ with a relative humidity of about $80.9 \%$. The water was slightly acidic (pH 6.62). We recorded T. anguliceps at Si Dong Yen, Chai Prakan district, Chiang Mai province, northern Thailand during
August in the same year $2014\left(19^{\circ} 38^{\prime} 31.8^{\prime \prime} \mathrm{N} 99^{\circ} 12^{\prime} 44.4^{\prime \prime} \mathrm{E}\right)$ at $1,260 \mathrm{~m}$ a.s.l. We found one adult male hiding under large rocks in a dry evergreen forest near a slow-flowing stream (Fig. 3B). The air temperature was $22.1{ }^{\circ} \mathrm{C}$ with a relative humidity of $73.2 \%$.

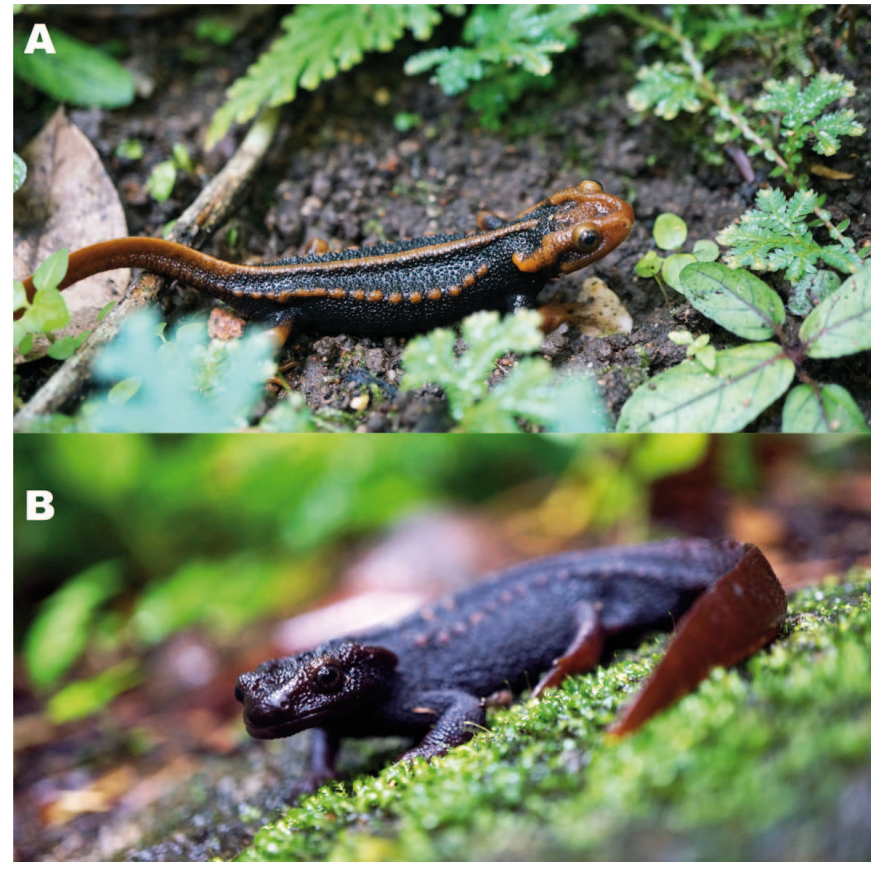

Figure 2. A. Adult male of T. uyenoi found at Doi Mon Jong, Tak province, north-western Thailand $\mathbf{B}$. Specimen of T. uyenoi at Doi Mak Lang ("group II" from Hernandez, 2016a,b) which is more related to T. verrucosus topotypic from Husa, Longchuan county, Yunnan province, China 


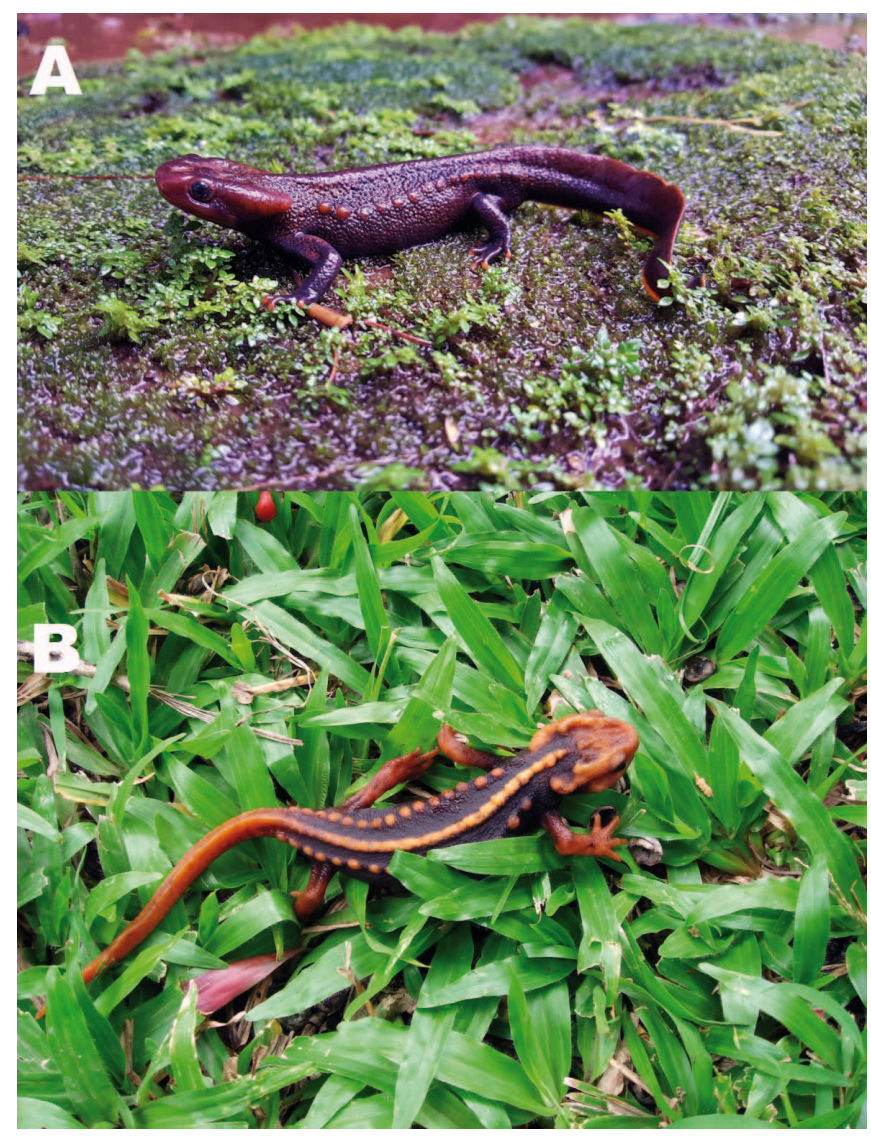

Figure 3. A. One male individual of T. panhai found in 2014 at Phu Thap Boek, Phetchabun province, north-eastern Thailand B. Specimen of $T$. anguliceps from Doi Lahnga range which is located $100 \mathrm{~km}$ south from our new locality in Si Dong Yen, Chiang Mai province, northern Thailand

\section{DISCUSSION}

These important new records confirm that crocodile newts of the genus Tylototriton inhabit mountainous moist evergreen forests at medium to high altitudes (Hernandez, 2016a,b, 2017; Hernandez et al., 2018). In the PCA, the first axis explained $54.0 \%$ of the variance and the second axis the $23.3 \%$. In the first axis the localities were segregated in a gradient of climate seasonality, precipitation and temperatures (factor loadings: temperature seasonality $=0.54$, precipitation seasonality $=$ 0.51 , mean annual precipitation $=0.53$ and mean temperature $=-0.37)$. In the second axis, the localities were segregated in a topographic and forest cover gradient (factor loadings: terrain ruggedness $=0.48$, forest cover $=0.70$ ), negatively associated with temperatures (mean temperature $=-0.46$ ). The PCA showed that these new sites were within the expected macroecological niche for these species, although in the case of $T$. uyenoi, these localities occupied two extremes within the environmental gradient (Fig. 4). However, macroecological models could fail to describe the niche of species that are closely linked to densely forested habitats (Scheffers et al., 2014); for this reason future studies should be directed to confirm this preliminary result, based on fine habitat characterisation. Moreover, the northernmost localities found in Thailand are Doi Mak Lang, Chiang Mai province for T. uyenoi (this paper), Phu Soi Dao, Uttaradit province for T. panhai (Hernandez, 2017) and Si Dong Yen for T. anguliceps (this paper). However, phylogenetic studies are needed to confirm the taxonomic status of these new populations. The Doi Mak Lang type differs in colour pattern and external morphology by having large cephalic edges, pronounced parotoid glands, skin finely granular, general ground colour dark brown above, glands on the neck and in the dorsolateral regions lighter brown to reddish orange. Indeed, Tylototriton species show a

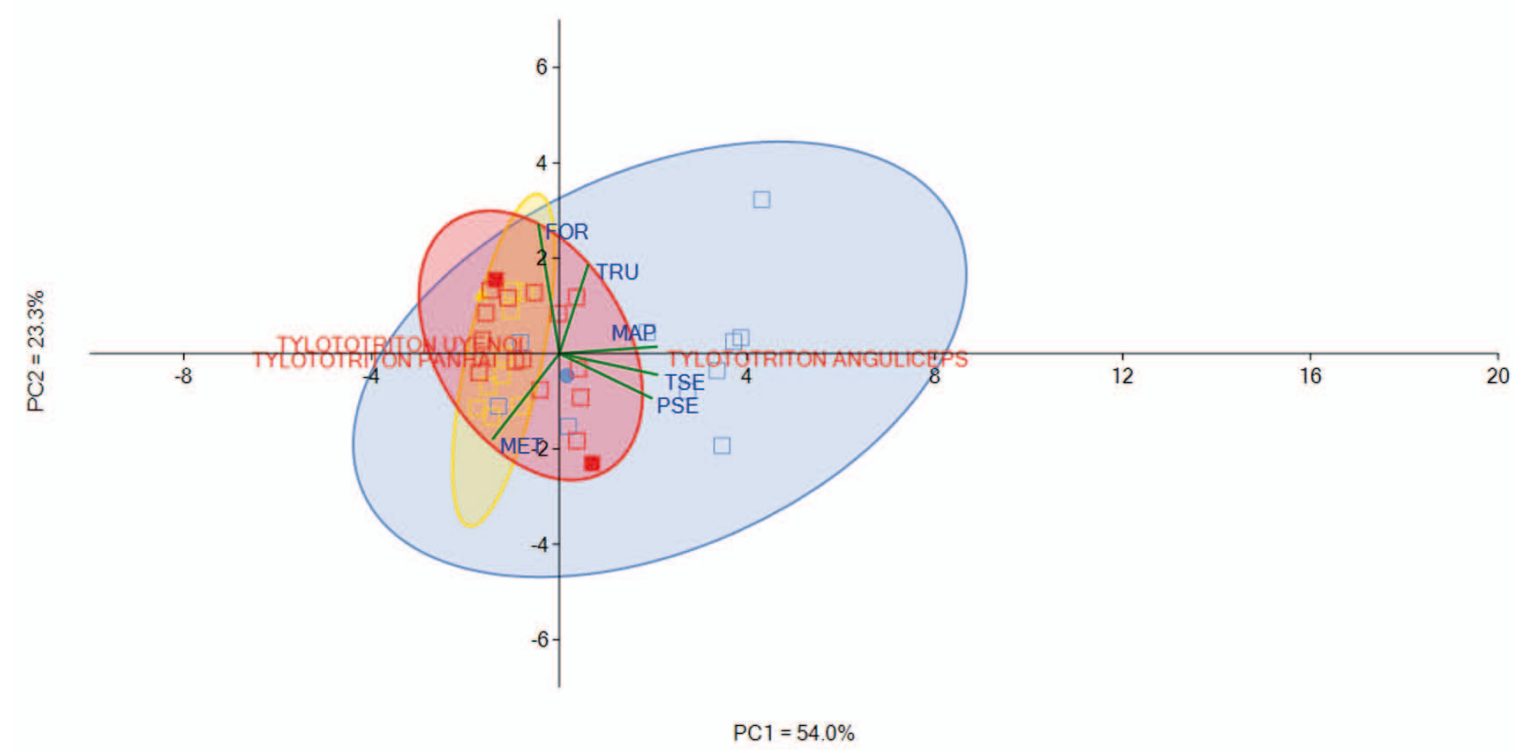

Figure 4. Crocodile newts ecological space, based on PCA. The $95 \%$ confidence ellipses are shown. FOR: Forest cover; MAP: Mean Annual Precipitation; MET: Mean annual temperature; PSE: Precipitation seasonality; TSE: Temperature seasonality: TRU: Terrain ruggedness. Blue, T. anguliceps; Red, T. uyenoi; Yellow, T. panhai. The new records are shown as filled figures, those described in Hernandez et al., 2008 as empty squares. 
conservative morphology that makes it difficult to differentiate between some species (Nishikawa et al., 2013; Le et al., 2015; Phimmachak et al., 2015). Genetic and ecological studies can provide new clues to classify these cryptic species (Hernandez et al., 2018).

Concerning T. panhai, we found three main phenotypes located in north-eastern Thailand (see Hernandez, 2016a,b) while for T. uyenoi, we found two main phenotypes including: (i) a northern and north-western type (including the type locality: Doi Suthep, Chiang Mai province); (ii) a north-eastern type which is similar to T. verrucosus sensu stricto. However, this latter group would require phylogenetic analyses to determine its true taxonomic affinities (Hernandez, 2015, 2016a,b, 2017). Regarding T. anguliceps we confirmed its presence in the three previously known localities in Thailand: Doi Lahnga, Doi Wiang Pha (Hernandez, 2015, 2016a; Le et al., 2015) and Si Dong Yen (this paper). All of these localities are within the same mountain range (pers. obs.). Furthermore, three other known localities of $T$. uyenoi are distributed through Tak province along the Dawana hills comprising: Doi Soi Malai, Umphang (Hernandez, 2015, 2016a,b, 2017) and Doi Mon Jong (this paper). These records constitute the southernmost localities for the species but also, for the whole genus Tylototriton extending $200 \mathrm{~km}$ southwards of its known distribution (Hernandez, 2017; Hernandez et al., 2018). Moreover, nearby mountains also have suitable habitats and could harbour undiscovered populations. More surveys are needed to improve knowledge about the distribution of these threatened crocodile newts and to establish conservation priorities

\section{ACKNOWLEDGEMENTS}

Thanks to Wichase Khonsue, Uwe Gerlach, Jean Raffaëlli, Emmanuel Jelsch and Jérome Maran. We also thank Vincent Descoteix for field survey and Thansuda Dowwiangkan for our useful discussion on Tylototriton. We also thank the National Park, Wildlife and Plant Conservation Department of Thailand (DNP), the Ministry of Natural Resources and Environment (MNRE), the Royal Forest Department of the Ministry of Agriculture and the Chulalongkorn University for support our study.

\section{REFERENCES}

Dowwiangkan, T., Ponpituk, Y., Chuaynkern, C., Chuaykern, Y. \& Duengkae, P. (2018). Population and habitat selection of Tylototriton uyenoi in Maesa-Kogma Biosphere Reserve, Chiang Mai Province, northern Thailand. Alytes 36: 300-313.

Gerlach, U. (2012). Im habitat das Birma-Krokodilmolches Tylototriton verrucosus in Thailand. Amphibia 11: 13-15.

Hammer, $\varnothing$. (2015). PAST: PAleontological STatistics software package, v3.06. Available at https://folk.uio.no/ ohammer/past/ (accessed January 2019)

Hernandez, A. (2015). Etude des Amphibiens Urodèles en voie d'extinction et mise en place de projets de conservation au Parc Zoologique de Thoiry. Master Thesis in Biology and Ecology. Università di Corsica Pasquale Paoli, Corte, France. 224 pp.
Hernandez, A. (2016a). Crocodile Newts, The Primitive Salamandridae from Asia (Genera Echinotriton and Tylototriton). Edition Chimaira. Germany. 415 pp.

Hernandez, A. (2016b). Tylototriton Anderson, 1871: Observation du genre en Thaïlande et éléments de réflexions sur la diversité des sous-genres Yaotriton et Tylototriton. Situla Revue Francophone d'Herpétoculture 28: $12-25$.

Hernandez, A. (2017). New localities for Tylototriton panhai and Tylototriton uyenoi Nishikawa, Khonsue, Pomchote \& Matsui, 2013 in northern Thailand. Bulletin la Société Herpétologique de France 162: 110-112.

Hernandez, A., Escoriza, D. \& Hou M. (2018). Patterns of niche diversification in south-east Asian crocodile newts. Zoologischer Anzeiger 276: 86-93.

Hijmans, R.J., Cameron, S.E., Parra, J.L., Jones, P.G., Jarvis, A. (2005). Very high resolution interpolated climate surfaces for global land areas. International Journal of Climatolology 25: 1965-1978.

Michaels, C.J. (2015). Field observations, morphometrics and a new locality for Tylototriton uyenoi in Chiang Mai Province, Thailand. Herpetological Bulletin 130: 26-27.

Le, D.T., Nguyen, T.T., Nishikawa, K., Nguyen, S.L.H., Pham, A.V., Matsui, M., Bernardes, M. \& Nguyen, T.Q. (2015). A new species of Tylototriton Anderson, 1871 (Amphibia: Salamandridae) from Northern Indochina. Current Herpetology 34 : 38-50.

Nabhitabhata, J. \& Chan-ard, T. (2005). Thailand Red Data: Mammals, Reptiles and Amphibians. Office of Natural Resources and Environmental Policy and Planning, Bangkok Thailand. $186 \mathrm{pp}$.

Nishikawa, K., Khonsue, W., Pomchote, P. \& Matsui, M. (2013). Two new species of Tylototriton from Thailand (amphibia: Urodela: Salamandridae). Zootaxa 3737: 261-279.

Phimmachak, S., Aowphol, A. \& Stuart, B. L. (2015). Morphological and molecular variation in Tylototriton (Caudata: Salamandridae) in Laos, with description of a new species. Zootaxa 4006: 285-310.

Pomchote, P., Pariyanonth, P., \& Khonsue, W. (2008). Two distinctive color patterns of the Himalayan newt Tylototriton verrucosus (Urodela: Salamandridae) found in Thailand and its implication on geographic segregation. The Natural History Journal of Chulalongkorn University 8: $35-43$.

Raffaëlli, J. (2013). Les Urodèles du Monde. Deuxième édition. Penclen editions. France, $480 \mathrm{pp}$.

Scheffers, B.R., Edwards, D.P., Diesmos, A., Williams, S.E., \& Evans, T.A. (2014). Microhabitats reduce animal's exposure to climate extremes. Global Change Biology 20: 495-503.

Taylor, E. H. (1962). The Amphibian fauna of Thailand. The University of Kansas Science Bulletin 43: 265-599.

Tuanmu, M.N. \& Jetz, W. (2014). A global 1-km consensus land-cover product for biodiversity and ecosystem modelling. Global Ecology and Biogeography 23: 10311045.

Wongratana, T. (1984). Range extension of the Crocodile salamander, Tylototriton verrucosus, to Phu Luang, Thailand. Natural History Bulletin of the Siam Society 32: 107-110.

Accepted: 09 January 2019 